$\underline{\text { Review Article }}$

\title{
NEUROPSYCHIATRIC ADVERSE EFFECTS OF ANTIBACTERIAL AGENTS
}

\author{
SANTA TREASA CYRIAC, DIVYA SARA IYPE \\ College of Pharmacy, PSG Institute of Medical Sciences \& Research, Coimbatore, Tamilnadu, India 641004 \\ Email: treesacyr@gmail.com
}

Received: 20 Jun 2021, Revised and Accepted: 26 Oct 2021

\begin{abstract}
Anti-bacterial are agents that inhibit bacterial growth or kills bacteria and are a sub-type of antimicrobials. These are drugs used to treat infections, but they sometimes pose a threat of adverse events. Some of these adverse events are neuropsychiatric, which are generally hard to diagnose and is often paid less attention. They account for about 30\% of total Adverse drug reactions (ADRs) caused by drugs in patients without mental abnormalities. The spectrum ranges from episodes of seizure to acute psychosis. The article emphasizes the frequency of such adverse events and means to raise awareness among medical practitioners regarding the same. The various neuropsychiatric adverse effects and the agents responsible have been reviewed, along with their possible mechanisms and general management.
\end{abstract}

The information for writing this review was selected by searching for keywords such as Neurotoxicity, GABA, Psychosis, Naranjo scale, and Antibiomania in databases such as Google Scholar, PubMed, Elsevier, etc. After searching the articles in the above-mentioned databases, the articles were screened concerning their importance with our work and according to their title and abstract. Additional articles were discovered by checking the references in the current study's citations. Using this method, the various neuropsychiatric adverse effects of Antibacterial agents were summarized in this review.

Keywords: Neurotoxicity, GABA, Psychosis, Naranjo scale, Antibiomania

(C) 2021 The Authors. Published by Innovare Academic Sciences Pvt Ltd. This is an open access article under the CC BY license (https://creativecommons.org/licenses/by/4.0/] DOI: https://dx.doi.org/10.22159/ijpps.2021v13i12.42482. Journal homepage: https://innovareacademics.in/journals/index.php/ijpps.

\section{INTRODUCTION}

Antibacterial drugs cover and possess many structural and functiona characteristics, and the case of one agent may be different from that of another [1]. While some of these agents are naturally derived, others are synthetic. These agents can be classified based on various features such as the mechanism of action, spectrum of the activity (bacteriostatic/bactericidal) etc. Here, we would use the structural classification of antibacterial agents to explain the neuropsychiatric adverse effects caused by them. The classes of importance mentioned in context are: Beta-lactams, Fluoroquinolones, Nitrofurans, Amino acids, Diaminopyridines, Sulfonamides, Oxazolidinones, Nicotinic acid derivatives, Nitroimidazoles, Tetracyclines, Antimycobacterial antibiotics, Macrolides, Sulfones, Nitrobenzene derivatives, Glycylcyclines, Lipopeptides, and Polypeptide antibiotics [1-6] Antimicrobial-related neurotoxic effects can have a vast array of presentations. Patients with focal sensory system illness, renal inadequacy and old age might be susceptible to these unwanted effects [2]. Conditions such as dizziness, tremors, hallucinations occur in 9$11 \%$ of patients on antibiotics [3]. Any antibiotic can cause an increased risk of depression [1]. Other changes in behavioural aspects range from insomnia [4] to acute psychosis [5]. Clarithromycin induced psychosis, for example, have been reported in $4-30 \%$ of patients; worst effects are seen on the nervous system in 3\% of patients which includes dizziness, anxiety, insomnia, bad dreams, confusion, disorientation and hallucination [6]. Commonly seen neuropsychiatric adverse drug effects upon administration of these antibacterials are seizures, psychosis, encephalopathy, peripheral neuropathy, optic neuropathy, worsening of myasthenia gravis, dizziness/vertigo, headache, and insomnia [1-6], which in detail have been discussed inside. Study publications, case reports and review articles of relevance with respect to the topic, published during 20042021 were referred to prepare this review.

Important neuropsychiatric adverse effects reported upon the use of anti-bacterial drugs

\section{Seizures}

Episodes are most commonly associated with antibiotic classes such as penicillin, cephalosporins, carbapenem and fluoroquinolones [2,

4, 7-13]. Other antibiotics associated with seizures include
Metronidazole, Nitrofurantoin, Cycloserine, Trimethoprimsulfamethoxazole (TMP-SMX), Linezolid, Tedizolid and an overdose of Isoniazid (INH) $[11,14,15]$. The risk of occurrence is higher with Imipenem compared to all other carbapenems [7].

Though mostly generalized tonic-clonic seizures are seen to be associated, cases of simple and complex partial seizures too have been reported [2]. An epileptic condition lasting for more than 30 min is known as Non-convulsive status epilepticus (NCSE), clinically manifested by an altered mental state and associated with continuous epileptiform activity on the encephalogram [10]. Fourthgeneration cephalosporin and cefepime have been frequently reported to cause NSCE. Seizures are mostly subclinical, the only clinical feature is a non-localizing encephalopathy, and ultimately Electroencephalogram (EEG) is required to make the diagnosis [2].

Administration of antimicrobials at doses higher than that recommended, age above 50 , renal insufficiency, conditions leading to alterations of blood-brain barrier such as the presence of a tumor, make patients susceptible to seizures [7]. Also, a higher incidence was seen when Penicillin or Cephalosporins were in association with Antibiotic-Associated Encephalopathy (AAE) [9]. As with fluoroquinolones, the risk is increased by concomitant use of Nonsteroidal Anti-inflammatory Drugs (NSAIDs) [11]. Some antibiotics act by increasing or decreasing plasma levels of Antiepileptic drugs (AEDs) administered to patients. Whereas certain drugs such as Betalactams and Quinolones could possess a proconvulsant activity and therefore induce seizures in patients with no history of epilepsy [10].

To treat seizures, the culprit drug must be discontinued. Management of NCSE involves use of anticonvulsants such as Benzodiazepines, Phenytoin and Valproic acid $[2,11]$

\section{Encephalopathy}

Antibiotics such as Metronidazole, Fluoroquinolones, Macrolides, Beta-lactams and Sulfonamides are most likely to cause encephalopathy $[2,7-11,16]$. The various features of AAE have been reviewed by Bhattacharya et al. Based on these features, encephalopathy has been categorized into three classes, Type 1 , Type 2 and Type 3 AAE. Encephalopathy beginning within days of antibiotic initiation, with the occurrence of myoclonus or seizures 
being common, disturbed EEG, normal Magnetic Resonance Imaging (MRI), which resolves within days are referred to as Type 1 AAE. Examples of antibiotics associated with Type $1 \mathrm{AAE}$ include penicillin and cephalosporins. Characteristics attributed to Type 2 AAE are commencement within days of initiation of therapy, psychotic episodes which occur frequently, the uncommon occurrence of seizures, infrequently abnormal EEG, normal MRI and resolution within days. Antibiotics commonly associated with Type 2 AAE are Procaine penicillin, Sulfonamides, Fluoroquinolones and Macrolides. Type 3 AAE can be attributed to encephalopathy induced by Metronidazole alone. The features include onset post a week of drug initiation, cerebellar dysfunction occurring frequently, rare seizures, rare yet non-specific EEG abnormalities and abnormal MRI [9].

EEG monitoring is recommended in patients who are being administered with antibiotics of neurotoxic potential. EEG is also helpful in distinguishing an episode of NCSE from encephalopathy. Once diagnosed, the culprit drug shall be replaced with an agent that is not neurotoxic [2].

\section{Psychosis}

It is the term used to describe illness in which patients have altered perception of reality as evidenced by delusion and/or hallucinations [17]. Psychosis, that is delusion and hallucinations were present in $47 \%$ of cases and were most commonly associated with Sulfonamides (68\%), Quinolones (67\%), Macrolide (63\%), and Penicillin IM (68\%) treatments. In fact, in medical literature, Psychosis is a secondary adverse effect caused by Clarithromycin. Psychosis was least seen in the case of AAE with Cephalosporins (13\%) and Metronidazole $(24 \%)[6,8]$.

The term used to describe an emergency manic episode in a reaction to antibiotics is 'antibiomania' or Hoigné syndrome $[8,18]$. It is a rare side effect shown by some antibiotics that resolve after cessation of the treatment. Macrolides and quinolones are comparatively more commonly associated, and at a lower rate are Beta-lactams and Metronidazole [1].

Several case reports and series provides in detail accounts of antibacterial-induced psychosis and their management. The case of Imipenem-cilastatin-induced psychosis by Jacob Ninan et al. describes an episode of acute psychosis upon an increase of the antibiotic dose. The patient experienced intense visual and auditory hallucinations due to Imipenem-cilastatin induced psychosis. According to the literature, the condition resolves in $2 \mathrm{w}$ of discontinuation of the causative agent [13].

A case of Metronidazole-induced psychosis has been discussed by Mili Khandheria et al. The manifestations (paranoia, delusions, auditory hallucinations) shown by the patient met the criteria for substance-induced psychosis as under the Diagnostic and Statistical Manual of Mental Disorders-IV (DSM-IV). The patient had been administering Metronidazole (500 mg twice daily). Psychosis resolved $2 \mathrm{~d}$ after withdrawal of the drug. The patient was prescribed Olanzapine ( $5 \mathrm{mg}$ twice daily) if her symptoms were to recur [3]. Withdrawal of the offending agents and administration of Diazepam injection produced an effect in the 28-year old male with INH and ethambutol induced psychosis, reported by Prasad R et al. [17]. We also see Risperidone being used to treat hallucinations caused by Trimethoprim-sulfamethoxazole which was prescribed for Urinary tract infection (UTI). The antibiotic, therefore, was substituted with Nitrofurantoin in this case report by Matej Stuhec [19]. Surprisingly, unlike these cases, Minocycline has been reported to produce improvements in patients with psychotic disorders. The drug has anti-inflammatory properties and is said to be capable of producing an effect on neurotransmitters as well as their receptors [20]. But it was also seen listed among antibiotics causing psychosis, and therefore cannot be ruled out completely off the picture [13].

An example of psychosis associated with type 2 AEE is Procaine penicillin (9). The cases show a relationship between psychosis and the administered antibiotic. However, in some cases, the underlying infectious condition itself (and not the antimicrobial) may be associated with exacerbation of psychosis. For example, the prevalence of comorbid infections during episodes of psychosis among schizophrenic patients, especially UTI are high [21]. A similar observation is the association between UTI and acute psychosis seen among geriatric patients [22]. Another relationship between infection and psychosis is marked by the increased risk of psychosis in an offspring whose mother experienced Genito-urinary infections or maternal fever during pregnancy [16].

Table 1: Drugs causing seizure, psychosis and encephalopathy

\begin{tabular}{|c|c|c|c|}
\hline Condition & Class of drugs & Drugs & References \\
\hline \multirow[t]{8}{*}{ Seizure } & Beta-lactams & $\begin{array}{l}\text { Penicillin, Piperacillin, Cefepime, Cefixime, Cefazolin, } \\
\text { Cefuroxime, Ceftazidime, Imipenem, Meropenem }\end{array}$ & {$[2,7-11,13]$} \\
\hline & Fluroquinolones & $\begin{array}{l}\text { Ciprofloxacin, Levofloxacin, Ofloxacin, Moxifloxacin, } \\
\text { Gatifloxacin, Norfloxacin }\end{array}$ & {$[2,4,7,11,12]$} \\
\hline & Nitrofurans & Nitrofurantoin & [14] \\
\hline & Amino acid & Cycloserine & [15] \\
\hline & Diaminopyridine-Sulfonamides & Trimethoprim-Sulfamethoxazole & [11] \\
\hline & Oxazolidinones & Linezolid, tedizolid & \\
\hline & Nicotinic acid derivatives & Isoniazid (on overdose) & \\
\hline & Nitroimidazoles & Metronidazole & \\
\hline \multirow[t]{10}{*}{ Psychosis } & Beta-lactams & $\begin{array}{l}\text { Penicillin, Procaine Penicillin, Procaine benzyl penicillin, } \\
\text { Piperacillin, Amoxicillin, Cefalexin, Meropenem, Imipenem }\end{array}$ & {$[1,8,9,11,13,16,21,22]$} \\
\hline & Fluroquinolones & $\begin{array}{l}\text { Ciprofloxacin, Ofloxacin, Norfloxacin, Temafloxacin, } \\
\text { Gatifloxacin, Levofloxacin, Moxifloxacin }\end{array}$ & {$[1,2,4,8,9,11-13,16,21-25]$} \\
\hline & Macrolides & Clarithromycin, Clindamycin, Erythromycin & {$[1,6,8,9,11,13,16,21]$} \\
\hline & Tetracyclines & Tetracycline, Doxycycline, Minocycline & {$[13,21]$} \\
\hline & Aminoglycosides & Amikacin, Gentamicin, & \\
\hline & Nitroimidazoles & Metronidazole & {$[1,3,8,9,11,13,21]$} \\
\hline & Diaminopyridine-Sulfonamide & Trimethoprim-Sulfamethoxazole & {$[1,2,8,9,11,13,16,19,22,26]$} \\
\hline & Nicotinic acid derivative & Isoniazid & {$[11,13,17]$} \\
\hline & Antimycobacterial antibiotics & Ethambutol & [17] \\
\hline & Amino acid & Cycloserine & [3] \\
\hline \multirow[t]{8}{*}{ Encephalopathy } & Beta-lactams & $\begin{array}{l}\text { Penicillin, Benzyl penicillin, Piperacillin, Procaine } \\
\text { penicillin, Cefepime, Ceftazidime, Cefazolin, Cefuroxime }\end{array}$ & {$[2,7,9-11,16]$} \\
\hline & Fluoroquinolones & Gemifloxacin & {$[2,7,9,11,16]$} \\
\hline & Macrolides & Clarithromycin & {$[7,9,11,16]$} \\
\hline & Nitroimidazole & Metronidazole & {$[2,7-9,11]$} \\
\hline & Diaminopyridine-Sulfonamide & Trimethoprim-Sulfamethoxazole, & {$[2,7,9,11,16]$} \\
\hline & Nicotinic acid derivative & Isoniazid (overdose) & [11] \\
\hline & Oxazolidinones & Linezolid & {$[2,11]$} \\
\hline & Aminoglycosides & Gentamicin & \\
\hline
\end{tabular}




\section{Peripheral neuropathy}

Drug-induced peripheral neuropathy takes place when damage to the nervous system has been caused by a chemical substance. It could be irreversible and may lead to paresthesia. Awareness among healthcare practitioners is important, especially when patients undergoing treatment with these drugs complain of pain, numbness, weakness, paresthesia or autonomic dysfunction. The duration required for the onset of these symptoms ranges from weeks to months [7, 27]. The various types of peripheral neuropathy are classified based on symptoms present - Sensory, motor, sensorimotor, and autonomic. It can also be classified based on pathophysiology or according to the type of nerve fibre affected. Once diagnosed, management requires discontinuation of the offensive agent and in the case of INH-induced peripheral neuropathy, Pyridoxine supplementation [11].

It is induced mainly by antibiotic drugs such as Fluoroquinolones, Metronidazole, Linezolid and INH [2, 3, 7, 11, 17, 24]. With INH, the most observed adverse effect concerning the neuropsychological context is peripheral neuropathy itself $[11,17]$. The onset of INHinduced peripheral neuropathy varies and may take up to 6 mo. higher doses of INH have been found to increase the risk of developing peripheral neuropathy. Concomitant intake of Pyridoxine is therefore advised [27]. Disease conditions that cause peripheral neuropathy, old age, malnutrition, and pregnancy are risk factors too [11].

As with Fluoroquinolones, in 2013 the Food and Drug Administration (FDA) wanted the label of Fluoroquinolones changed to highlight the risk of peripheral neuropathy $[28,11]$. Later in 2014, in a study of 6,226 cases and 24,904 controls, conducted by Mahyar Etminan et al., the risk of peripheral neuropathy with oral Fluoroquinolones were analyzed. The results revealed a risk ratio of 1.83 among current users and 2.07 among current new users [28].

The incidence is as high as $50 \%$ with Metronidazole and Linezolid, when these drugs are used for a long duration, or when Metronidazole is used concomitantly with Selective serotonin reuptake inhibitors (SSRIs) [7, 2]. Metronidazole-induced peripheral neuropathy may be present alone or in association with Metronidazole induced encephalopathy (MIE). Linezolid-induced optic neuropathy is often preceded by peripheral neuropathy, which may continue despite the withdrawal of the offending agent and resolution of symptoms of the eye $[2,11]$.

Polymyxin, Telavancin, Daptomycin, Linezolid and Nitrofurantoin are the agents that account for paresthesia. It is recommended that Linezolid shall be administered only for a maximum duration of $28 \mathrm{~d}$ as it may cause paresthesia of extremities of the body. Nitrofurantoin-related peripheral neuropathy is said to begin as paresthesia of distal extremities, which later develops dysesthesia. Other antibiotics capable of causing peripheral neuropathy are Dapsone, Chloramphenicol, Gentamicin, Ethambutol, and Sulfasalazine $[2,7,11]$, as shown in table 2 .

\section{Optic neuropathy}

Antibiotics have also been found to cause optic neuropathy. The causative agents include many antibiotics such as Ethambutol, Linezolid, Ciprofloxacin, Levofloxacin, Chloramphenicol, Metronidazole, INH, Streptomycin and Sulfonamides. Ethambutol and Linezolid were the most commonly reported agents. Manifestations associated with optic neuropathy include painless, progressive loss of vision of both eyes. There could be a reduction in colour vision, i.e., the patient may fail to discriminate between red and green. Optic neuropathy induced by antibiotics is often reversible, which means the patient returns to normal once the drug is withheld $[2,7,11]$.

Linezolid-induced optic neuropathy can be identified by some additional features, such as optic disc swelling or pallor, loss of central vision (more than that of peripheral vision). It is reversible in nature. When compared to Linezolid, a better alternative would be Tedizolid since it shows a much lower risk towards the development of optic neuropathy [11].

Risk factors associated with Ethambutol-induced optic neuropathy are prolonged therapy, higher doses, old age (above $60 \mathrm{y}$ ), hypertension and renal dysfunction. The onset of the condition may vary from 1-9 mo. The nature of Ethambutol-induced optic neuropathy may be irreversible at times. It can cause permanent loss of vision, though it is unlikely to occur in most patients. In cases where it is reversible, improvement is seen within 3 mo. [11]

Table 2: Drugs causing peripheral neuropathy, optic neuropathy and exacerbation of myasthenia gravis

\begin{tabular}{|c|c|c|c|}
\hline Condition & Class of drugs & Drugs & References \\
\hline Peripheral & Oxazolidinone & Linezolid & {$[2,7,11,27]$} \\
\hline \multirow[t]{11}{*}{ Neuropathy } & Sulfones & Dapsone & [7] \\
\hline & Nitrobenzene derivatives & Chloramphenicol & \\
\hline & Sulfonamides & Sulfasalazine & \\
\hline & Aminoglycosides & Gentamicin, & {$[2,11]$} \\
\hline & Fluroquinolones & Ciprofloxacin, Levofloxacin, Moxifloxacin & {$[7,11,24,28]$} \\
\hline & Lipopeptides & Daptomycin & [11] \\
\hline & Lipoglycopeptide & Telavancin & \\
\hline & Nicotinic acid derivative & Isoniazid & {$[7,11,17,27]$} \\
\hline & Nitrofurans & Nitrofurantoin & {$[7,11]$} \\
\hline & Nitroimidazoles & Metronidazole & [27] \\
\hline & Antimycobacterial antibiotics & Ethambutol & {$[7,11,27]$} \\
\hline \multirow[t]{7}{*}{ Optic neuropathy } & Oxazolidinones & Linezolid & {$[2,7,11]$} \\
\hline & Fluoroquinolones & Ciprofloxacin, Levofloxacin & [7] \\
\hline & Nitrobenzene derivatives & Chloramphenicol & \\
\hline & Sulfonamides & - & \\
\hline & Aminoglycosides & Streptomycin & \\
\hline & Nitroimidazoles & Metronidazole & {$[2,7]$} \\
\hline & Nicotinic acid derivatives & Isoniazid & {$[7,11]$} \\
\hline Exacerbation of & Fluoroquinolones & - & [7] \\
\hline \multirow[t]{5}{*}{ Myasthenia gravis } & Macrolides & Clindamycin & \\
\hline & Aminoglycosides & - & \\
\hline & Tetracyclines & - & \\
\hline & Polypeptide antibiotics & Colistin, Polymyxin-B & {$[2,7]$} \\
\hline & Beta-lactam & $\begin{array}{l}\text { Ampicillin, Ceftolozane, Ceftaroline, } \\
\text { Doripenem. Imipenem }\end{array}$ & {$[2,7,11]$} \\
\hline
\end{tabular}

'-'indicates that a specific agent was not mentioned among the given references. 


\section{Exacerbation of myasthenia gravis}

This event has been reported with the use of Polymyxins such as Colistin and Polymyxin B. Other than this, Aminoglycosides, Fluoroquinolones, Macrolides, Tetracyclines, Ampicillin, and Imipenem are the antibiotics implicated $[2,7,11]$. Myasthenia gravis is an autoimmune disorder. Clinical manifestations of Myasthenia gravis are diplopia, ptosis, dysarthria, dysphagia, proximal limb weakness. These symptoms are worsened by activity or towards the end of the day. The exacerbation of this condition could be of seriousness, stretching between mild to severe. When severe, the patient may suffer from respiratory failure and therefore require ventilatory support, as denoted by the term 'myasthenic crises'. Polymyxin injections may lead to a respiratory failure lasting 10-48 h. Close monitoring of symptoms is recommended in patients with myasthenia gravis, taking antibiotic medications $[2,7,12]$.

\section{Headache}

Fluoroquinolones, Beta-lactams, Oxazolidinones, Sulfonamides, Lipoglycopeptides, Clarithromycin, Polymyxins, Tigecycline, Minocycline, Daptomycin, Nitrofurantoin, INH, Rifampicin, Metronidazole and Dalfopristin-quinupristin, have been reported to cause headache $[2,4,11,12,23,24,29]$.

It is a common side effect seen with Sulfonamides [11]. Among Beta-lactams, headache is said to be common with the use of Doripenem and Ceftaroline [2]. A mild headache could be accompanied by Rifampin administration. Among Oxazolidinones, Linezolid and Tedizolid had headache listed among their most common side effects during the ESTABLISH trial [11]. Clinical trials with Dalbavancin shows that the major neurological side effect associated with the drug is headache, which was experienced by $25 \%$ of the subjects [30]. Dalbavancin, as well as
Telavancin (another Lipoglycopeptide), are drugs of importance in cases of complicated skin and skin structure infections where Vancomycin fails to produce effect [31]. The safety of Telavancin was assessed in a randomized comparative study conducted by Michael W. Dunne et al. A total of 1778 patients were enrolled. A dose of $500 \mathrm{mg}$ was administered on the first day and $1000 \mathrm{mg}$ on the $8^{\text {th }}$ day for treatment of skin and skin structure infections. The results of the study mention headache as one of the important side effects associated with this drug [32].

Apart from these, Streptogramins are agents whose usage has been limited to the treatment of infections caused by Vancomycinresistant enterococci (VRE). Headache is the only neuropsychiatric adverse event seen with their use [2].

\section{Dizziness/vertigo}

Dizziness and vertigo are conditions that occur as a result of vestibular toxicity [11]. In the case review by Serafina Chimirri et al., Dizziness has been defined as a general term used to express subjective complaints of the patient in connection with the changes in sensation, movement, perception or consciousness. Vertigo, however, is a subtype of dizziness and has been defined as an illusion of movement caused by asymmetric involvement of the vestibular system [33]. As mentioned in table 3, Fluoroquinolones, Polymyxins, Clarithromycin, Metronidazole, Minocycline, Tigecycline, Daptomycin, Gentamicin, Vancomycin, Linezolid, Nitrofurantoin, Rifampin and Ethambutol [2, 4, $6,11,12,17,23,24,29,33$ ] are the agents found to be associated with Dizziness/Vertigo.

The case report by Boonsong Kiangkitiwan et al. briefs an incident of levofloxacin-induced delirium with psychotic features where the 42 year old female patient experienced neuropsychiatric adverse effects, including dizziness [4].

Table 3: Drugs causing headache, dizziness/vertigo and insomnia

\begin{tabular}{|c|c|c|c|}
\hline Condition & Class of drugs & Drugs & References \\
\hline \multirow{15}{*}{ Headache } & Macrolides & Clarithromycin & {$[11,29]$} \\
\hline & Fluoroquinolones & Ofloxacin, Gemifloxacin, Ciprofloxacin, Moxifloxacin & {$[2,4,11,12,23,24]$} \\
\hline & Polypeptide antibiotics & Polymyxin & {$[11]$} \\
\hline & Glycylcyclines & Tigecycline & \\
\hline & Oxazolidinones & Linezolid, Tedizolid & \\
\hline & Tetracyclines & Minocycline & \\
\hline & Sulfonamides & - & \\
\hline & Lipopeptides & Daptomycin & \\
\hline & Nitrofurans & Nitrofurantoin & \\
\hline & Nicotinic acid derivatives & Isoniazid & \\
\hline & Antimycobacterial antibiotics & Rifampin & \\
\hline & Nitroimidazoles & Metronidazole & {$[2]$} \\
\hline & Streptogramins & Dalfopristin-quinupristin & \\
\hline & Lipoglycopeptides & Dalbavancin, Telavancin, Oritavancin & {$[11,30-32]$} \\
\hline & Beta-lactams & Ceftolozane, Ceftaroline & {$[11,34]$} \\
\hline \multirow[t]{13}{*}{ Dizziness/vertigo } & Fluoroquinolones & $\begin{array}{l}\text { Ciprofloxacin, Ofloxacin, Moxifloxacin, Gemifloxacin, } \\
\text { Levofloxacin }\end{array}$ & {$[2,4,11,12,23,24,33]$} \\
\hline & Nitroimidazoles & Metronidazole & {$[2]$} \\
\hline & Macrolides & Clarithromycin & {$[6,29]$} \\
\hline & Polypeptide antibiotics & Polymyxin & {$[2,11]$} \\
\hline & Tetracyclines & Minocycline & [11] \\
\hline & Glycylcyclines & Tigecycline & \\
\hline & Lipopeptides & Daptomycin & \\
\hline & Aminoglycosides & Gentamycin & \\
\hline & Glycopeptides & Vancomycin & \\
\hline & Oxazolidinones & Linezolid & \\
\hline & Nitrofurans & Nitrofurantoin & \\
\hline & Antimycobacterial antibiotics & Rifampin, Ethambutol & {$[11,17]$} \\
\hline & Beta-lactams & Amoxicillin-clavulanic acid & [33] \\
\hline \multirow[t]{9}{*}{ Insomnia } & Glycylcyclines & Tigecycline & [11] \\
\hline & Lipopeptides & Daptomycin & \\
\hline & Oxazolidinones & Linezolid & \\
\hline & Nicotinic acid derivatives & Isoniazid & \\
\hline & Polypeptide antibiotics & Polymyxin B & \\
\hline & Fluoroquinolones & Levofloxacin, Moxifloxacin & {$[2,11,24]$} \\
\hline & Lipoglycopeptides & Dalbavancin, Telavancin & {$[11,32]$} \\
\hline & Beta-lactams & Ceftolozane & {$[11,34]$} \\
\hline & Macrolides & Azithromycin, Clarithromycin & {$[6,29]$} \\
\hline
\end{tabular}

'-'indicates that a specific agent was not mentioned among the given references. 


\section{Insomnia}

It refers to the difficulty initiating or maintaining sleep, or both. Antibiotics causing insomnia are Fluoroquinolones, Cephalosporins, Macrolides, Tigecycline, Dalbavancin, Telavancin, Daptomycin, Linezolid, INH [2, 4, 6, 11, 12, 23, 29, 32].

In a case series consisting of 3 individual cases published by Arun Kandasamy et al., we see patients with lower respiratory tract infections who underwent Levofloxacin therapy. The patients experienced insomnia which was caused by Levofloxacin itself. The first case is that of a 30-year old male patient who took levofloxacin $500 \mathrm{mg}$ for $5 \mathrm{~d}$ on a once-daily basis. Another case is that of a 30-year old female patient who took Levofloxacin $500 \mathrm{mg}$ daily. The last case is that of a patient who selfmedicated himself with a $750 \mathrm{mg}$ dose of Levofloxacin. All three patients had their symptoms of anxiety and insomnia resolved once Levofloxacin was stopped. None of the patients took drugs other than Levofloxacin, nor did any show history of psychiatric illness. Thus, Levofloxacin was the offending agent here [12].

Mechanisms involved in the causation of neuropsychiatric adverse effects by various anti-bacterial agents:

For antibiotics associated with psychotic abnormalities such as Fluoroquinolones, Cephalosporins, Penicillins, and Trimethoprimsulfamethoxazole drugs, several possible mechanisms have been proposed. There is proof as assessed, that anti-infectives can cause intense responses such as seizures. The drugs reaching harmful amounts in the bloodstream, their anti-inflammatory properties and their ability to inhibit prostaglandin E-2, or cause Gamma-aminobutyric acid (GABA) antagonism, and N-methyl-D-aspartate (NMDA) receptor hypo functioning are a few of the possible mechanisms. The last two factors have generally been discussed as mechanisms that serve as the basis for Schizophrenic disorder [8]. Investigations are suggesting that Quinolones such as Levofloxacin cause acute anxiety and insomnia by antagonism of the inhibitory GABA and direct activation of excitatory NMDA receptors. The structural similarity between Fluoroquinolones and GABA could be held reason for their antagonistic activity. They also act directly on Alphaamino-3-hydroxy-5-methyl-4-isoxazole propionic acid (AMPA) receptors. These mechanisms are connected to the non-dopaminergic pathways for the development of psychosis $[12,21,24]$. In addition to these, the moderately lipophilic nature of Fluoroquinolones increases their ability to cross the blood-brain barrier [21]. The effect produced by Benzodiazepines and Barbiturates are effective treatment strategies in cases of Cephalosporin-induced epileptiform activity that supports the possibility of GABA blockade by Cephalosporins to cause neurotoxicity [10].

GABA class A receptor (GABAAR) of stellate cells of the cerebellar region is controlled by the level of mitochondrial reactive oxygen species (mROS) at inhibitory synapses. Some antibiotics which are likely to target pathogens will also bind with mitochondria of brain cells, for example, Minocycline causing antimicrobial-induced mania. Changes in behaviour could be attributed to the use of drugs such as Ciprofloxacin, Metronidazole, Ofloxacin, TMP-SMX, Cotrimoxazole, Procaine penicillin and Clarithromycin, as GABA receptor binding could be inhibited by these drugs [25]. The pathway involving Levofloxacin-incited delirium stays to be explained. It is conceivable that Quinolone-related NCSE is responsible, asQuinolones are known to bring down the seizure edge by competitively binding to the GABAAR [4]. Macrolides and Quinolones are the classes commonly prone to antibiomania. The mechanism underlying this condition is hypothesized to be caused by GABA antagonism, antibiotic-induced mitochondrial dysfunction, Cytochrome P450 3A4 (CYP3A4) inhibitory effect or abnormalities with the microbiota. In any case, the use of antibiotics is most certainly associated with infectious and immune-related disorders [1, 21].

The mechanism involved in Type1 AAE is the inhibition of ligand-gated ion channel, which is also linked with GABAAR. Type 2 is thought to be associated with Dopamine abnormalities and the NMDA pathway. The last type, type 3 might be due to free radical formation and alterations which may occur in Thiamine metabolism [8].

The Tetracycline drugs, such as Minocycline and Doxycycline, have immunoregulatory as well as anti-inflammatory properties. They also possess good Central nervous System-penetration characteristics. These drugs have been found to produce antiinflammatory effects in in vivo and in vitro conditions, both. They may therefore act on neurons and glia [1]. The ability of the drug to penetrate and reach toxic levels has been inflicted in TMP-SMX related hallucinations as well [19]. Impairment in metabolism and reduced clearance of the drug adds to the challenge [26].

INH causes increased excretion of Pyridoxine, resulting in this vitamin's deficiency. As a result, the usual Tryptophan metabolism gets disturbed. Brain pyridoxal-5-phosphate too is inhibited by this antibiotic since this coenzyme is produced from pyridoxine itself. Inhibition of the enzyme results in a decrease in GABA of the brain and other synaptic transmitters. Thus neurological adverse effects are elicited. Conditions such as diabetes mellitus, hepatic insufficiency, old age, alcoholism, and family and personal history of mental illness predisposes the patient towards INH-induced neuropsychiatric adverse effects [17, 27].

Another mechanism suggested to comply with the case report published by Mili Khandheria et al., is that the enzyme, monoamine oxidase (MAO) is reversibly inhibited by Metronidazole. The enzyme involved in the Dopamine pathway is responsible for its breakdown. Thus a decrease in MAO results in excess Dopamine. Since the inhibition is reversible, this explanation backs the fact that the patient recovered after Metronidazole was stopped [3]. Another possible explanation for Metronidazole-induced neurotoxicity, which involves cytotoxic and vasogenic oedema was also put forward [21].

\section{Evaluation and management}

Diagnostic criteria for psychiatric disorders are mentioned in DSMIV [17]. The Diagnostic and Statistical Manual of Mental Disorders-V (DSM-V) was used to confirm psychosis induced by Imipenemcilastatin in the case report by Jacob Ninan et al. [13]. Naranjo adverse drug reaction probability scale (table 4) has helped in the assurance of ADRs in many cases referred; A score over 9 demonstrates definite ADR while 5-8 means a probable ADR. Scores 1-4 insights possible ADR, and zero a doubtful ADR $[9,13,19,24,29]$. EEG and MRI could be of use in cases of AAE and seizures [2, 9]. A strategy commonly used to confirm substance-induced psychosis is by ensuring the resolution of symptoms with the withdrawal of the drug and their recurrence on rechallenge with the same agent $[2,11$, $13,22,35,36]$. However, performing a rechallenge may not be justifiable at all times [29]. Challenges to the diagnosis of Fluoroquinolone-related neurotoxicity include False-positive urineopiate immunoassay reports caused by their ability to cross-react with the Enzyme immunoassay (EIA) screens for opiate drugs [4].

Table 4: Naranjo adverse drug reaction probability scale

\begin{tabular}{|c|c|c|c|c|}
\hline Question & Yes & No & $\begin{array}{l}\text { Do not } \\
\text { know }\end{array}$ & Score \\
\hline 1. Are there previous conclusive reports on this reaction? & +1 & 0 & 0 & \\
\hline 2. Did the adverse event appear after the suspected drug was administered? & +2 & -1 & 0 & \\
\hline 3. Did the adverse reaction improve when the drug was discontinued or a specific antagonist was administered? & +1 & 0 & 0 & \\
\hline 4. Did the adverse reaction reappear when the drug was re-administered? & +2 & -1 & 0 & \\
\hline 5. Are there alternative causes (other than the drug) that could on their own have caused the reaction? & -1 & +2 & 0 & \\
\hline 6. Did the reaction reappear when a placebo was given? & -1 & +1 & 0 & \\
\hline 7. Was the drug detected in blood (or other fluids) in concentrations known to be toxic? & +1 & 0 & 0 & \\
\hline 8. Was the reaction more severe when the dose was increased or less severe when the dose was decreased? & +1 & 0 & 0 & \\
\hline 9. Did the patient have a similar reaction to the same or similar drugs in any previous exposure? & +1 & 0 & 0 & \\
\hline $\begin{array}{l}\text { 10. Was the adverse event confirmed by any objective evidence? } \\
\text { Total Score }\end{array}$ & +1 & 0 & 0 & \\
\hline
\end{tabular}


Naranjo ADR Probability Scale: A method for estimating the probability of adverse drug reactions. Clin Pharmacol Ther. 1981 [37].

A close association is often seen between the administration of antibiotics and the occurrence of adverse events. Once the condition is diagnosed, the primary step in management would be to withdraw the causative agent and substitute it with a non-neurotoxic antibiotic. In many a case, this leads to the cessation of the adverse effect. In contrast to this, rare cases like that of Nitrofurantoininduced dysesthesias may cause irreversible damage. This infers that the withdrawal of the drug does not undo the harm caused. With some antibiotics, however, adverse effects are caused in a dose-dependent fashion, in such cases, dosage adjustments are necessitated [2, 38-51]. It may not be practical to completely withdraw the causative agent at all times though [39].

Treatment of the condition may be performed alongside if necessary. A case of secondary mania may be treated with antipsychotics, benzodiazepines and mood stabilizers. The use of second-generation antipsychotics is considered better in comparison with the firstgeneration. The initial steps to manage seizure would be stabilization of the airways, maintenance of blood pressure and pulse, measurement of serum glucose levels and management of hyperthermia. In the case of NCSE, anticonvulsants may be required for treatment. Other forms of epilepsy or tremors shall be treated with Anti-epileptic drugs. Myasthenic syndrome caused by polymyxin may have to be aided with ventilatory support. It is decided based on the degree of respiratory impairment $[2,39,52-60]$.

If the renal function is impaired and diagnosis of antibiotic-induced neurotoxicity is confirmed, haemodialysis or hemofiltration may have to be performed to ensure adequate clearance of the drug. A high-volume continuous venovenous hemofiltration (CVVHF) can be used to optimize drug clearance. Drugs with a low tendency towards protein binding can easily be controlled by CVVHF. Medication doses should be cautiously adjusted in patients with renal dysfunction [2].

Conditions such as higher prevalences of nosocomial infections and cases of antimicrobial resistance in Intensive care unit-like settings complicates the threat further [61].

\section{CONCLUSION}

We would recommend the usage of antibiotics to be performed strictly, as per protocols. Since neurotoxic impacts of antimicrobials are hard to perceive, their occurrence is regularly disparaged. It turns out to be significantly difficult in patients with meningitis-like neurological ailments. Lasting side effects may lead to further complications. Given the globally wide utilization of antimicrobial agents like Amoxicillin, for example, neuropsychiatric complications can't be overlaid. Consequently, there is a requirement for expanded watchfulness during treatment with such medicines. Regular monitoring should be warranted whenever culprit drugs are employed in the treatment, particularly when higher doses are used. Awareness about antibiotics associated with neurotoxic side effects may improve overall healthcare. The maintenance of a proper database could help in the identification of the population at risk, quicker diagnosis and treatment. Of equal importance is Hospital based-intensive monitoring, which has proved to be a great way of detecting relationships between drug exposure and consequent adverse drug reactions. Diligent vigilance is required to find out potential neurotoxic effects and to provide patients with optimum therapy. ADRs reduce the quality of life and therefore, treatment should always aim at improving it. Strong vigilance and the data implicated helps in increasing the knowledge of health professionals in these areas. Pharmacists should therefore participate in clinical rounds and documentation procedures to ensure safe use of these drugs.

\section{FUNDING}

Nil

\section{AUTHORS CONTRIBUTIONS}

All the authors have contributed equally.

\section{CONFLICT OF INTERESTS}

Declared none

\section{REFERENCES}

1. Champagne Jorgensen K, Kunze WA, Forsythe P, Bienenstock J, McVey Neufeld KA. Antibiotics and the nervous system: More than just the microbes? Brain Behav Immun. 2019;77:7-15. doi: 10.1016/j.bbi.2018.12.014, PMID: 30582961.

2. Grill Marie F, Maganti Rama K. Neurotoxic effects associated with antibiotic use: management considerations. $\mathrm{Br} \mathrm{J}$ Clin Pharmacol. 2011;72(3):381-93. doi: 10.1111/j.13652125.2011.03991.x, PMID 21501212.

3. Khandheria Mili, Snook Erica, Thomas Christopher. Psychotic episode secondary to metronidazole use. Gen Hosp

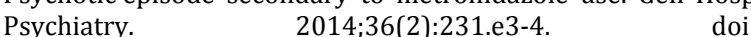
10.1016/j.genhosppsych.2013.11.009, PMID 24388629.

4. Kiangkitiwan Boonsong, Doppalapudi Avanthi, Fonder Margaret, Solberg Kim, Bohner Brian. Levofloxacin-induced delirium with psychotic features. Gen Hosp Psychiatry. 2008;30(4):381-3. doi: 10.1016/j.genhosppsych.2007.11.003, PMID 18585545.

5. Przybylo HJ, Przybylo Jason H, Todd Davis A Todd, Cote Charles J. Acute psychosis after anesthesia: the case for antibiomania. Paediatr Anaesth. 2005;15(8):703-5. doi: 10.1111/j.14609592.2004.01525.x, PMID 16029408.

6. Elahi Fawad, Bhamjee Moosajee. A case of clarithromycin psychosis. Psychiatr Bull. 2004;28(3):98-9. doi: 10.1192/pb.28.3.98.

7. Bhattacharyya Shamik, Darby Ryan, Berkowitz Aaron L. Antibiotic-induced neurotoxicity. Curr Infect Dis Rep. 2014;16(12):448. doi: 10.1007/s11908-014-0448-3, PMID 25348743.

8. Ly Duy, DeLisi Lynn E. Can antibiotics cause a psychosis?: case report and review of the literature. Schizophr Res. 2017;189:204-7. doi: 10.1016/j.schres.2017.01.041, PMID 28185785.

9. Bhattacharya Shamik, Ryan Darby R, Raibagkar Pooja, Nicolas Gonzalez Castro L, Berkowitz Aaron L. Antibiotic-associated encephalopathy. American Academy of Nursing; 2016.

10. Anzellotti F, Ricciardi L, Monaco D, Ciccocioppo F, Borrelli I, Zhuzhuni H, Onofrj M. Cefixime-induced nonconvulsive status epilepticus. Neurol Sci. 2012;33(2):325-9. doi: $10.1007 / \mathrm{s} 10072-011-0668-3$. PMID 21720898.

11. Bangert Madison K, Hasbun Rodrigo. Neurological and psychiatric adverse effects of antimicrobials. CNS Drugs. 2019;33(8):727-53. doi: 10.1007/s40263-019-00649-9, PMID 31321707.

12. Kandasamy Arun, Srinath D. Levofloxacin-induced acute anxiety and insomnia. J Neurosci Rural Pract. 2012;3(2):212-4 doi: 10.4103/0976-3147.98256, PMID 22865986.

13. Ninan Jacob, George Gemy Maria. Imipenem-cilastatin-induced psychosis: a case report. J Med Case Rep. 2016;10(1):107. doi: 10.1186/s13256-016-0883-x, PMID 27118305.

14. Stuhec Matej, Svab Vesna. Nitrofurantoin-induced lifethreatening seizures. Wien Klin Wochenschr. 2012;124(1718):653-4. doi: 10.1007/s00508-012-0220-3, PMID 22850815.

15. Fujita Junichi, Sunada Kouichi, Hayashi Hiroki, Hayashihara Kenji, Saito Takefumi. A case of multi-drug resistant tuberculosis showing adverse psychiatric effects by cycloserine. Kekkaku. 2008;83(1):21-5. PMID 18283911.

16. Klein Petersen Amalie W, Köhler Forsberg Ole, Benros Michael E. Infections, antibiotic treatment and the microbiome in relation to schizophrenia. Schizophr Res. 2021;234:71-7. doi: 10.1016/j.schres.2019.11.033, PMID 31859119.

17. Prasad R, Garg Rajiv, Verma Sanjay Kumar. Isoniazid- and ethambutol-induced psychosis. Ann Thorac Med. 2008;3(4):14951. doi: 10.4103/1817-1737.43083, PMID 19561898.

18. Legendre T, Boudebesse C, Henry C, Etain B. Antibiomania: think of the manic syndrome secondary to antibiotic therapy. Encephale. 2017;43(2):183-6. doi: 10.1016/j.encep. 2015.06.008, PMID 27178256.

19. Stuhec Matej. Trimethoprim-sulfamethoxazole-related hallucinations. Gen Hosp Psychiatry. 2014;36(2):230.e7-8. doi: 10.1016/j.genhosppsych.2013.10.016, PMID 24268951.

20. Çakici N, van Beveren NJM, Judge-Hundal G, Koola MM, Sommer IEC. An update on the efficacy of anti-inflammatory agents for 
patients with schizophrenia: a meta-analysis. Psychol Med. 2019;49(14):2307-19. doi: 10.1017/S0033291719001995, PMID 31439071.

21. Essali Norah, Miller Brian J. Psychosis as an adverse effect of antibiotics. Brain Behav Immun Health. 2020;9:100148. doi: 10.1016/j.bbih.2020.100148, PMID 34589893.

22. Mostafa Safinaz, Miller Brian J. Antibiotic-associated psychosis during treatment of urinary tract infections: a systematic review. J Clin Psychopharmacol. 2014;34(4):483-90. doi: 10.1097/JCP.0000000000000150, PMID 24911441.

23. Rossi Garrett, Mazoki Kristen. Acute psychosis after treatment of epididymitis with ciprofloxacin. Cureus. 2018;10(5):e2605. doi: 10.7759/cureus.2605, PMID 30013869.

24. Mazhar Faizan, Akram Shahzad, Haider Nafis. Moxifloxacininduced acute psychosis: A case report with literature review. J Res Pharm Pract. 2016;5(4):294-6. doi: 10.4103/2279042X.192457, PMID 27843968.

25. Stefano George B, Samuel Joshua, Kream Richard M. Antibiotics may trigger mitochondrial dysfunction inducing psychiatric disorders. Med Sci Monit. 2017;23:101-6. doi: 10.12659/msm.899478, PMID 28063266.

26. Saidinejad Mohsen, Ewald Michele Burns, Shannon Michael W. Transient psychosis in an immune-competent patient after oral trimethoprim-sulfamethoxazole administration. Pediatrics. 2005;115(6):e739-41. doi: 10.1542/peds.2004-1352, PMID 15867013.

27. Jones Mark R, Urits Ivan, Wolf John, Corrigan Devin, Colburn Luc, Peterson Emily, Williamson Amber, Viswanath Omar. Drug-induced peripheral neuropathy: A narrative review. Curr Clin Pharmacol. 2020;15(1):38-48. doi: 10.2174/ 1574884714666190121154813 , PMID 30666914.

28. Etminan Mahyar, Brophy James M, Samii Ali. Oral fluoroquinolone use and risk of peripheral neuropathy: a pharmacoepidemiologic study. Neurology. 2014;83(14):12613. doi: 10.1212/WNL.0000000000000846, PMID 25150290.

29. Farooq Osman, Memon Zebunnissa, Stojanovski Sasko D, Faden Howard S. Azithromycin-induced agitation and choreoathetosis. Pediatr Neurol. 2011;44(4):311-3. doi: 10.1016/j.pediatrneurol.2010.11.014, PMID 21397177.

30. Leighton Anton, Gottlieb Alice Bendix, Dorr Mary Beth, Jabes Daniela, Mosconi Giorgio, VanSaders Claudia, Mroszczak Edward J, Campbell Kathleen CM, Kelly Ellen. Tolerability, pharmacokinetics, and serum bactericidal activity of intravenous dalbavancin in healthy volunteers. Antimicrob Agents Chemother. 2004;48(3):940-5. doi: 10.1128/AAC.48.3.940-945.2004, PMID 14982787.

31. Zhanel George G, Divna Calic, Frank Schweizer, Sheryl Zelenitsky, Hether Adam, Philippe RS Lagace Wiens. New lipoglycopeptides: a comparative review of dalbavancin, oritavancin and telavancin. Drugs 2010;70:859-86. doi: 10.2165/11534440-000000000-00000, PMID: 20426497

32. Dunne Michael W, Talbot George H, Boucher Helen W, Wilcox Mark, Puttagunta Sailaja. Safety of dalbavancin in the treatment of skin and skin structure infections: A pooled analysis of randomized, comparative studies. Drug Saf. 2016;39(2):147-57. doi: 10.1007/s40264-015-0374-9, PMID 26715497

33. Serafina Chimirri Serafina, Rossana Aiello Rossana, Carmela Mazzitello Carmela, Laura Mumoli Laura, Caterina Palleria Caterina, Mariolina Altomonte Mariolina, Citraro Rita, De Sarro Giovambattista. Vertigo/dizziness as a Drugs' adverse reaction. J Pharmacol Pharmacother. 2013;4(Suppl 1):s104-9S104-9. doi: 10.4103/0976-500X.120969, PMID 24347974.

34. Maria Sorbera Maria, Elizabeth Chung Elizabeth, Christopher W, Ho Christopher W, Nino Marzella Nino. Ceftolozane/tazobactam: a new option in the treatment of complicated gram-negative infections. P T. 2014;39(12):82532. PMID 25516692.

35. Christopher Jones S, Sorbello Alfred, Boucher Robert M. Fluoroquinolone-associated myasthenia gravis exacerbation: evaluation of postmasketing reports from the US FDA adverse event reporting system and a literature review. Drug Saf. 2013;34:839-47. https://doi.org/10.2165/11593110000000000-00000
36. Krishnarao A, Feller E, Shah S. Metronidazole-induced mania: recurrence with Re-challenge 924. Am J Gastroenterol. 2011;106. doi: 10.14309/00000434-201110002-00924.

37. Naranjo CA, Busto U, Sellers EM, Sandor P, Ruiz I, Roberts EA, Janecek E, Domecq C, Greenblatt DJ. A method for estimating the probability of adverse drug reactions. Clin Pharmacol Ther. 1981;30(2):239-45. doi: 10.1038/clpt.1981.154, PMID 7249508.

38. Ik Lin Tan, Michael J Polydefkis, Gigi J Ebenezer, Peter Hauer, Justin C McArthurTan Ik Lin, Polydefkis Michael J, Ebenezer Gigi J, Hauer Peter, McArthur Justin C. Peripheral nerve toxic effects of nitrofurantoin Peripheral nerve toxic effects of nitrofurantoin. Arch Neurol. 2012;69(2):265-8. doi: 10.1001/archneurol.2011.1120, PMID 22332195.

39. Simon Lambrichts Simon, Lukas van Oudenhove Lukas, Pascal Sienaert Pascal. Antibiotics and mania: a systematic review. J Affect Disord. 2017;219:149-56. doi: 10.1016/j.jad.2017.05.029, PMID 28550767.

40. SJ Martin SJ, FJ Bowden FJ. Ethambutol toxicity manifesting as acute onset psychosis. Int J STD AIDS. 2007;18(4):287-8. doi: 10.1258/095646207780658863, PMID 17509184.

41. Piyush Puri Piyush, Pankul Parnami Pankul, Akshit Chitkara Akshit, Pal Satyajit Singh Athwal Pal Satyajit Singh, Sunil Khetrapal Sunil. Antibiomania: A rare case of metronidazoleinduced mania. Cureus. 2021;13(1):e12414. doi: 10.7759/cureus.12414, PMID 33409111.

42. Megan K. Skelly Megan K, Bethany A, Wattengel Bethany A, Kaitlyn E, Starr Kaitlyn E, John A, Sellick John A, Kari A, Mergenhagen Kari A. Psychiatric adverse effects of antibiotics. Psychiatr. Times; 2019.

43. Steinert T, Studemund H. Acute delusional parasitosis under treatment with ciprofloxacin. Pharmacopsychiatry. 2006;39(4):159-60. doi: 10.1055/s-2006-947183, PMID 16871474.

44. Nicholas H, Neufeld Nicholas H, Nourhan S, Mohamed Nourhan S, Nikola Grujich Nikola, Kenneth Shulman Kenneth. Acute neuropsychiatric symptoms associated with antibiotic treatment of helicobacter pylori infections: a review. J Psychiatr Pract. 2017;23(1):25-35. doi: 10.1097/PRA.0000000000000205, PMID 28072642.

45. J Babbar J, T Foster T. Brief psychosis associated with triple therapy for peptic ulcer. Internet J Fam Pract. 2005;4.

46. Mitanshu ShahShah Mitanshu, Subhani Miral, Rizvon Kaleem, Mustacchia Paul, Miral Subhani, Kaleem Rizvon, Paul Mustacchia. Transient psychotic episode induced by helicobacter pylori triple therapy treatment. Case Rep Gastroenterol. 2012;6(2):381-6. doi: 10.1159/000339713, PMID 22855656.

47. Navid Khalili Navid. Sunny hypomania associated with clarithromycin: a case report. J Clin Psychopharmacol. 2014;34(3):416-7. doi: 10.1097/JCP.0000000000000107, PMID 24717249.

48. Nicholas Zareifopoulos Nicholas, George Panayiotakopoulos George. Neuropsychiatric effects of antimicrobial agents. Clin Drug Investig. 2017;37(5):423-37. doi: 10.1007/s40261-0170498-z, PMID 28197902.

49. Cilia Nazef Cilia, Aazim Arif Aazim. Ciprofloxacin-induced acute psychosis: a case report. Am J Med Case Rep. 2019;7(7):143-4. doi: 10.12691/ajmcr-7-7-6

50. Susanna Esposito Susanna, Maria Paola Canevini Maria Paola, Nicola Principi Nicola. Complications associated with antibiotic administration: neurological adverse events and interference with antiepileptic drugs. Int J Antimicrob Agents. 2017;50(1):18. doi: 10.1016/j.ijantimicag.2017.01.027, PMID 28414069.

51. Kuan Yeh Lee, Chung Hao Huang, Hung Jen Tang, Chia Jui Yang, Wen Chien Ko, Yen Hsu Chen, Lee Yi-Chien, Hung Chien Ching. Acute psychosis related to use of trimethoprim/sulfamethoxazole in the treatment of HIV-infected patients with Pneumocystis jirovecii pneumonia: a multicentre, retrospective study. J Antimicrob Chemother. 2012;67(11):2749-54. doi: 10.1093/jac/dks283, PMID 22821870.

52. Eduard B Dinca Eduard B, Alex Skinner Alex, Ramona V Dinca Ramona V, Catalina Tudose Catalina. The dangers of gastritis: a case of clarithromycin-associated brief psychotic episode. J Nerv Ment Dis. 2015;203(2):149-51. doi: 10.1097/ NMD.0000000000000251, PMID 25629665. 
53. Zahiruddin O, Shanooha M, Mohd Azhar MY. Psychosis induced by cefuroxime and metronidazole. Med J Malaysia. 2014;69(1):33-4. PMID 24814627.

54. Pitchaya Wanleenuwat Pitchaya, Nanthushan Suntharampillai Nanthushan, Piotr Iwanowski Piotr. Antibiotic-induced epileptic seizures: mechanisms of action and clinical considerations. Seizure. 2020;81:167-74. doi: 10.1016/j.seizure.2020.08.012, PMID 32827980.

55. Yibin Guo Yibin, Xiaojiao Shao, Liyuan Zhang Liyuan, Jiayi Miao Jiayi, Yiwen Zhang Yiwen. A case of suspected antibioticassociated encephalopathy in a patient undergoing long-term peritoneal dialysis. Int J Med Res. 2020;48:1-6. doi: 10.1177/0300060520924507, PMID: 32459125

56. Ajay Kumar Bakhla Ajay Kumar, Prakash S Gore Prakash S, Sanjay Lal Srivastava Sanjay Lal. Cycloserine-induced mania. Ind Psychiatry J. 2021;22(1):69-70. doi: 10.4103/09726748.123636, PMID 24459378.

57. Shree Bhalerao Shree, Aaron Talsky Aaron, Keith Hansen Keith, Edward Kingstone Edward, Ben Schroeder Ben, Zamil Karim Zamil, Fung Irene. Ciprofloxacin-induced manic episode.
Psychosomatics. 2006;47(6):539-40

doi: 10.1176/appi.psy.47.6.539, PMID 17116962.

58. Yuk-Fai Cheung, Winnie Wing-Yin Wong, Kwok-Wing Tang, John Hiu-Ming Chan, Patrick Chung-Ki LiCheung Yuk-Fai, Wong Winnie Wing-Yin, Tang Kwok-Wing, Chan John Hiu-Ming, Li Patrick ChungKi. Ciprofloxacin-induced palatal tremor. Mov Disord. 2007;22(7):1038-43. doi: 10.1002/mds.21452, PMID 17357133.

59. Weis S, Karagülle D, Kornhuber J, Bayerlein K. Cotrimoxazoleinduced Psychosis: A case report and review of the literature. Pharmacopsychiatry. 2006;39(6):236-7. doi: 10.1055/s-2006950393, PMID 17124650.

60. Oliver Grimm Oliver, Barbara Alm Barbara, Fur Seelische Zentralinstitut. A case of ciprofloxacin-induced acute polymorphic psychosis with a distinct deficit in executive functions. Psychosomatics. 2007;48(3):269. doi: 10.1176/appi.psy.48.3.269, PMID 17478598.

61. Al-zakwani Ibrahim, Al-thuhli Marwa, Al-Hashim Abdulhakeem, Al Balushi KA. Drug utilization pattern in an intensive care unit at a teritiary care teaching hospital in Oman. Asian J Pharm Clin Res. 2017;10:194-7. DOI: 10.1177/0300060520924507, PMID: 32459125 